(C) 1998 International Press

Adv. Theor. Math. Phys. 2 (1998) 1011-1040

\title{
Vanishing of intersection numbers on the moduli space of
} Higgs bundles

\author{
Tamás Hausel \\ School of Mathematics \\ Institute for Advanced Study \\ Olden Lane, Princeton \\ New Jersey 08540, USA \\ hausel@math.ias.edu
}

\begin{abstract}
In this paper we consider the topological side of a problem which is the analogue of Sen's S-duality testing conjecture for Hitchin's moduli space $\mathcal{M}$ of rank 2 stable Higgs bundles of fixed determinant of odd degree over a Riemann surface $\Sigma$. We prove that all intersection numbers in the compactly supported cohomology of $\mathcal{M}$ vanish, i.e. "there are no topological $L^{2}$ harmonic forms on $\mathcal{M}$ ". This result generalizes the well known vanishing of the Euler characteristic of the moduli space of rank 2 stable bundles $\mathcal{N}$ of fixed determinant of odd degree over $\Sigma$. Our proof shows that the vanishing of all intersection numbers of $H_{c p t}^{*}(\mathcal{M})$ is given by relations analogous to the Mumford relations in the cohomology ring of $\mathcal{N}$.
\end{abstract}

\section{Introduction}

Analyzing the conjectured S-duality in $\mathrm{N}=2$ supersymmetric Yang-Mills theory, which is a proposed $S L(2, \mathbb{Z})$ symmetry of the theory, Sen in [Sen] could predict the dimension of the space of $L^{2}$ harmonic forms $\mathcal{H}_{k}$ on the universal cover of the moduli space of magnetic monopoles of charge $k$, by speculating

e-print archive: http://xxx.lanl.gov/abs/math.AG/9805071 
that there must be an $S L(2, \mathbb{Z})$ action on the space $\bigoplus \mathcal{H}_{k}$, which represents bound electron states of the theory.

The moduli space of monopoles $M_{k}$ of charge $k$ is the space of finite energy and charge $k$ solutions to the Bogomolny equations in $\mathbb{R}^{3}$, which can be interpreted as a reduction of the self-dual $S U(2)$ Yang-Mills equations in $\mathbb{R}^{4}$. The space $M_{k}$ is a non-compact manifold, with $\pi\left(M_{k}\right)=\mathbb{Z}_{k}$, and has a natural hyperkähler and complete metric on it, which comes from an abstract construction (the so-called hyperkähler quotient construction, cf. [HKLR]) and known explicitly only in the case $k=2$, when $M_{2}$ is called the Atiyah-Hitchin manifold. (For further details see [At,Hi].)

When $k=2$ Sen's conjecture says that $\operatorname{dim}\left(\mathcal{H}_{2}\right)=1$. By knowing the metric of $M_{2}$ explicitly, Sen was able to find a non-trivial $L^{2}$ harmonic form on the universal cover $\tilde{M}_{2}$, giving some support for his conjecture and in turn for S-duality.

For higher $k$ Sen's conjecture says something about a metric which is not known explicitly. Nevertheless the statement is interesting from a mathematical point of view as the space of $L^{2}$ harmonic forms on a non-compact complete Riemannian manifold is not well understood.

Hodge theory tells us that in the compact case the space of $L^{2}$ harmonic forms is naturally isomorphic to the De-Rham cohomology of the manifold. However in the non-compact case there is no such theory, and indeed the harmonic space depends crucially on the metric.

Nevertheless some part of Hodge theory survives for complete Riemannian manifolds (cf. [DeRh] Sect. 32 Theorem 24 and Sect. 35 Theorem 26), such as the Hodge decomposition theorem which states that for a complete Riemannian manifold $M$ the space $\Omega_{L^{2}}^{*}$ of $L_{2}$ forms on $M$ has an orthogonal decomposition

$$
\Omega_{L^{2}}^{*}=\overline{d\left(\Omega_{c p t}^{*}\right)} \oplus \mathcal{H}^{*} \oplus \overline{\delta\left(\Omega_{c p t}^{*}\right)}
$$

and also $\mathcal{H}^{*}=\operatorname{ker}(d) \cap \operatorname{ker}\left(d^{*}\right)$.

An easy corollary ${ }^{1}$ of these results says that the composition

$$
H_{c p t}^{*}(M) \rightarrow \mathcal{H}^{*} \rightarrow H^{*}(M)
$$

is the forgetful map.

By calculating the image of $H_{c p t}^{*}\left(\tilde{M}_{k}\right)$ in $H^{*}\left(\tilde{M}_{k}\right)$ Segal and Selby could give a lower bound for the harmonic forms on the moduli space of magnetic

${ }^{1} \mathrm{Cf} .[\mathrm{Se}, \mathrm{Se}]$ 
monopoles which coincides with the dimension given by Sen's conjecture (see $[\mathrm{Se}, \mathrm{Se}])$. This purely mathematical result is thus a supporting evidence for the conjectured S-duality in $N=2 \mathrm{SYM}$ of theoretical Physics.

In this paper we will investigate the analogue of Sen's conjecture for Hitchin's moduli space $\mathcal{M}$ of rank 2 Higgs bundles of fixed determinant of degree 1 over a Riemann surface $\Sigma$ of genus $g>1$. The space $\mathcal{M}$ is a simply connected non-compact manifold of dimension $12 g-12$ with a complete hyperkähler metric on it, and was constructed by Hitchin in [Hit1] by considering the solutions of the self-dual Yang-Mills equations on $\mathbb{R}^{4}$ which are translation invariant in two directions. Led by the similarities between the spaces $M_{k}$ and $\mathcal{M}$ and their origin, we ask the following question:

Problem 1 What are the $L^{2}$ harmonic forms on $\mathcal{M}$ ?

In this paper we prove the following:

Theorem 1.1 The forgetful $m a p^{2}$

$$
j_{\mathcal{M}}: H_{c p t}^{*}(\mathcal{M}) \rightarrow H^{*}(\mathcal{M})
$$

is 0 .

This says that unlike the case of $\tilde{M}_{k}$ the topology of $\mathcal{M}$ does not give the existence of $L^{2}$ harmonic forms. We can state this fact informally as: "There are no topological $L^{2}$ harmonic forms on Hitchin's moduli space of Higgs bundles".

Segal and Selby's result together with Sen's conjecture suggest that for $\tilde{M}_{k}$ the topology gives all the harmonic space. Led by this and supported by the discussion in Subsection 2.2 we can formulate the following conjecture:

Conjecture 1 There are no non-trivial $L^{2}$ harmonic forms on Hitchin's moduli space of Higgs bundles.

It would be interesting to see whether a physical argument could back this conjecture. We know of one serious appearance of Hitchin's moduli space of Higgs bundles in the Physics literature. In [BJSV] a topological $\sigma$ model with target space $\mathcal{M}$ arises as certain limit of $N=4$ supersymmetric

\footnotetext{
${ }^{2}$ Unless otherwise stated cohomology is meant with real coefficients.
} 
Yang-Mills theory. However it is not clear whether $L^{2}$ harmonic forms on $\mathcal{M}$ have any physical interpretation in this theory.

Note that the conjecture does not hold for parabolic Higgs bundles, as the toy example in Example 2 after Theorem 7.13 of [Hau1] shows. Note also that Dodziuk's vanishing theorem [Dod] shows that there are no non-trivial $L^{2}$ holomorphic forms on $\mathcal{M}$, since the Ricci tensor of a hyperkähler metric is zero.

From an algebraic geometrical point of view Theorem 1.1 can be interpreted as follows. First of all it is really about middle dimensional cohomology, because it is known that $\mathcal{M}$ does not have cohomology beyond the middle dimension, and equivalently by Poincaré duality $\mathcal{M}$ does not have compactly supported cohomology below the middle dimension. Thus the main content of Theorem 1.1 is the vanishing of the canonical map $j_{\mathcal{M}}: H_{c p t}^{6 g-6}(\mathcal{M}) \rightarrow H^{6 g-6}(\mathcal{M})$ between $g$ dimensional spaces (cf. Corollary 5.4 in [Hau1]). This in turn is equivalent to the vanishing of the intersection form on $H_{c p t}^{6 g-6}(\mathcal{M})$, i.e. to the vanishing of $g^{2}$ intersection numbers.

There are $g+1$ intersection numbers whose vanishing follows easily. One vanishing is obtained by recalling that the moduli space $\mathcal{N}$ of stable bundles of real dimension $6 g-6$ sits inside $\mathcal{M}$ with normal bundle $T_{\mathcal{N}}^{*}$, thus its selfintersection number is its Euler characteristic up to sign, which is known to vanish.

The other $g$ vanishings follow from the fact that the ordinary cohomology class of the Prym variety, the generic fibre of the Hitchin map, is 0 , i.e. it is in the kernel of $j_{\mathcal{M}}$. This can be seen by thinking of the Hitchin map as a section of the trivial rank $3 g-3$ vector bundle on $\mathcal{M}$ and considering the ordinary cohomology class of the Prym variety as the Euler class of this trivial vector bundle, and as such, the ordinary cohomology class of the Prym variety is trivial indeed. Note that for the case $g=2$, the above vanishings are already enough to have $j_{\mathcal{M}}=0$. (Cf. Example 2 after Theorem 7.13 in [Hau1].)

The vanishing of the rest of the $g^{2}$ intersection numbers on $\mathcal{M}$, proved in the present paper for any genus, can be considered as a generalization of these facts.

The structure of this paper is as follows: In the next section we describe the cohomology of certain moduli spaces. In Section 3 we define hypercohomology groups and related notions. Then in Section 4 we develop the theory of stable Higgs bundles analogously to the stable vector bundle case, and prove an important vanishing theorem. In Section 5 we prove that $\tilde{\mathcal{M}}$ is 
a fine moduli space, and define certain universal bundles. In Section 6 we construct the virtual Dirac bundle as the analogue of the virtual Mumford bundle, and show that it can be considered as the degeneracy sheaf of a homomorphism of vector bundles. In Section 7 we determine the degeneracy locus of the above homomorphism in terms of the components of the nilpotent cone. Finally in Section 8 we prove our main Theorem 1.1 using Porteous' formula for the degeneracy locus of the virtual Dirac bundle.

Acknowledgements. First of all I would like to thank my supervisor Nigel Hitchin for suggesting Problem 1, and for his help and encouragement. I am grateful to Michael Thaddeus for his inspiring paper [Tha1], enlightening communications and his constant interest in my work. I am also indebted to Manfred Lehn for the idea of the proof of Theorem 6.2. I have found conversations with Michael Atiyah, Frances Kirwan and Graeme Segal very stimulating. I thank the Mathematical Institute and St. Catherine's College, Oxford for their hospitality during the preparation of this work. Finally I thank Trinity College, Cambridge for financial support.

\section{Moduli spaces and their cohomology}

The central object of this paper is a fixed, smooth and complex projective curve $\Sigma$ of genus $g \geq 2$. We also fix a point $p \in \Sigma$.

An additive basis of $H^{*}(\Sigma): 1 \in H^{0}(\Sigma), e_{i} \in H^{1}(\Sigma), i=1, . ., 2 g$ and the fundamental cohomology class $\sigma \in H^{2}(\Sigma)$ with the properties that $e_{i} \wedge e_{i+g}=$ $-e_{i+g} \wedge e_{i}=\sigma$ for $i=1, . ., g$ and otherwise $e_{i} \wedge e_{j}=0$, will be fixed throughout this paper.

\subsection{The Jacobian $\mathcal{J}$}

The moduli space of line bundles of degree $k$ over $\Sigma$ is the Jacobian $\mathcal{J}_{k}$. This is an Abelian variety of dimension $g$. Tensoring by a fixed line bundle of degree $k-l$ gives an isomorphism between $\mathcal{J}_{l}$ and $\mathcal{J}_{k}$. We will write $\mathcal{J}$ for $\mathcal{J}_{1}$.

Being a torus $H^{*}\left(\mathcal{J}_{k}\right)$ is a free exterior algebra on $2 g$ classes $\tau_{i} \in H^{1}\left(\mathcal{J}_{k}\right)$ defined by the formula

$$
c_{1}\left(\mathbb{L}_{k}\right)=k \otimes \sigma+\sum_{i=1}^{2 g} \tau_{i} \otimes e_{i} \in H^{2}\left(\mathcal{J}_{k} \times \Sigma\right) \cong \sum_{r=0}^{2} H^{r}\left(\mathcal{J}_{k}\right) \otimes H^{2-r}(\Sigma) .
$$


Here $\mathbb{L}_{k}$ is the normalized Poincaré bundle, or universal line bundle over $\mathcal{J}_{k} \times \Sigma$. Universal means that for any $L \in \mathcal{J}_{k}$ :

$$
\left.\mathbb{L}_{k}\right|_{\{L\} \times \Sigma} \cong L
$$

and normalized means that $\left.\mathbb{L}_{k}\right|_{\mathcal{J}_{k} \times\{p\}}$ is trivial (cf. [ACGH]).

\subsection{Moduli space of Abelian Higgs bundles $T_{\mathcal{J}}^{*}$}

As a toy example for the discussions in the Introduction, we consider here the moduli space of Abelian Higgs bundles.

The tangent bundle of $\mathcal{J}$ is canonically isomorphic to $\mathcal{J} \times H^{1}\left(\Sigma, \mathcal{O}_{\Sigma}\right)$. Thus by Serre duality $T_{\mathcal{J}}^{*} \cong \mathcal{J} \times H^{0}(\Sigma, K)$ canonically. An element $\Phi \in$ $\left(T_{\mathcal{J}}^{*}\right)_{L} \cong H^{0}(\Sigma, K)$, can be thought of as a rank 1 Higgs bundle: $\mathcal{L}=L \stackrel{\Phi}{\rightarrow}$ $L \otimes K$ (cf. Definition 4.1). Thus we can think of $T_{\mathcal{J}}^{*}$ as the moduli space of rank 1 Higgs bundles.

The cohomology of $T_{\mathcal{J}}^{*}$ is isomorphic to that of $\mathcal{J}$. However there is an extra piece of cohomological information namely the intersection numbers in the compactly supported cohomology or in other words the map:

$$
j_{\mathcal{J}}: H_{c p t}^{*}\left(T_{\mathcal{J}}^{*}\right) \rightarrow H^{*}\left(T_{\mathcal{J}}^{*}\right)
$$

Clearly this map is interesting only in the middle dimension, where both $H_{c p t}^{2 g}\left(T_{\mathcal{J}}^{*}\right)$ and $H^{2 g}\left(T_{\mathcal{J}}^{*}\right)$ are one-dimensional. However the Euler characteristic of $\mathcal{J}$ is clearly 0 , thus the self-intersection number of the zero section of $T_{\mathcal{J}}^{*}$ is 0 , which shows that $j_{\mathcal{J}}$ vanishes.

Consider the Riemann metric on $T_{\mathcal{J}}^{*} \cong \mathcal{J} \times H^{0}(\Sigma, K)$ which is the product of the flat metrics on the two terms (this is the metric which we get if we perform Hitchin's work in [Hit1] for the Abelian case). From the $L^{2}$ vanishing theorem of Dodziuk [Dod], since the metric is flat there are no non-trivial $L^{2}$ harmonic forms on $T_{\mathcal{J}}^{*}$, thus in the Abelian Higgs case the topology gives the harmonic space, as conjectured for the rank 2 Higgs moduli space in Conjecture 1 and for the universal cover of the moduli space of magnetic monopoles in [Sen].

\subsection{The moduli space of rank 2 stable bundles $\mathcal{N}$}

We denote by $\tilde{\mathcal{N}}$ the fine moduli space of rank 2 stable bundles with degree 1 over $\Sigma$. It is a smooth projective variety of dimension $4 g-3$. The determinant gives a map $\operatorname{det}_{\mathcal{N}}: \tilde{\mathcal{N}} \rightarrow \mathcal{J}$. For any $\Lambda \in \mathcal{J}$ the fibre $\operatorname{det}_{\mathcal{N}}^{-1}(\Lambda)$ 
will be denoted by $\mathcal{N}_{\Lambda}$, which is a smooth projective variety of dimension $3 g-3$. The map $f: \mathcal{N}_{\Lambda_{1}} \rightarrow \mathcal{N}_{\Lambda_{2}}$ given by $f(E)=E \otimes\left(\Lambda_{2} \otimes \Lambda_{1}^{*}\right)^{1 / 2}$, where $\left(\Lambda_{2} \otimes \Lambda_{1}^{*}\right)^{1 / 2}$ is a fixed square root of $\Lambda_{2} \otimes \Lambda_{1}^{*}$, is an isomorphism between $\mathcal{N}_{\Lambda_{1}}$ and $\mathcal{N}_{\Lambda_{2}}$. Hence we will write $\mathcal{N}$ for $\mathcal{N}_{\Lambda}$, when we do not want to emphasize the fixed line bundle $\Lambda$.

Let $G:=H^{1}\left(\Sigma, \mathbb{Z}_{2}\right) \cong \mathbb{Z}^{2 g} \cong \operatorname{ker}\left(\sigma_{2}\right)$, where $\sigma_{2}: \mathcal{J}_{0} \rightarrow \mathcal{J}_{0}$ is given by $\sigma_{2}(L)=L^{2}$. Now $G$ acts on $\mathcal{N}$ and $\mathcal{J}$ by tensoring with the corresponding line bundle in $\operatorname{ker}\left(\sigma_{2}\right)$ and also on $\mathcal{N} \times \mathcal{J}$ by the diagonal action. Then (cf. (9.5) of $[\mathrm{At}, \mathrm{Bo}])$ we have

$$
\tilde{\mathcal{N}}=(\mathcal{N} \times \mathcal{J}) / G
$$

Because $G$ acts trivially on $H^{*}(\mathcal{J})$ and on $H^{*}(\mathcal{N})$ (the latter was first proved in $[\mathrm{Ha}, \mathrm{Na}])$ we see that as rings

$$
H^{*}(\tilde{\mathcal{N}}) \cong\left(H^{*}(\mathcal{N}) \otimes H^{*}(\mathcal{J})\right)^{G} \cong H^{*}(\mathcal{N}) \otimes H^{*}(\mathcal{J})
$$

Thus for understanding the cohomology ring $H^{*}(\tilde{\mathcal{N}})$ it is enough to know the cohomology ring $H^{*}(\mathcal{N})$. The latter is multiplicatively generated by classes $\alpha_{\mathcal{N}} \in H^{2}(\mathcal{N}), \psi_{\mathcal{N}}^{i} \in H^{3}(\mathcal{N})$ and $\beta_{\mathcal{N}} \in H^{4}(\mathcal{N})$, which appear in the Künneth decomposition of $c_{2}\left(\operatorname{End}\left(\mathbb{E}_{\mathcal{N}}\right)\right)$ :

$$
c_{2}\left(\operatorname{End}\left(\mathbb{E}_{\mathcal{N}}\right)\right)=2 \alpha_{\mathcal{N}} \otimes \sigma+\sum_{i=1}^{2 g} 4 \psi_{\mathcal{N}}^{i} \otimes e_{i}-\beta_{\mathcal{N}} \otimes 1
$$

in $H^{4}(\mathcal{N} \times \Sigma) \cong \sum_{r=0}^{4} H^{r}(\mathcal{N}) \otimes H^{4-r}(\Sigma)$.

Here $\mathbb{E}_{\mathcal{N}}$ is the normalized rank 2 universal bundle over $\mathcal{N} \times \Sigma$, i.e. $c_{1}\left(\mathbb{E}_{\mathcal{N}}\right)=\alpha_{\mathcal{N}}$ and $\left.\mathbb{E}_{\mathcal{N}}\right|_{\{E\} \times \Sigma} \cong E$ for every $E \in \mathcal{N}$.

The ring $H^{*}(\mathcal{N})$ is described in terms of the so called Mumford relations. To explain this consider the virtual Mumford bundle

$$
\begin{aligned}
\mathrm{M}=-\pi_{\tilde{\mathcal{N}} !}\left(\mathbb{E}_{\tilde{\mathcal{N}}} \otimes \pi_{\Sigma}^{*}\left(L_{p}^{-1}\right)\right)= & -R^{0} \pi_{\tilde{\mathcal{N}} *}\left(\mathbb{E}_{\tilde{\mathcal{N}}} \otimes \pi_{\Sigma}^{*}\left(L_{p}^{-1}\right)\right) \\
& +R^{1} \pi_{\tilde{\mathcal{N}} *}\left(\mathbb{E}_{\tilde{\mathcal{N}}} \otimes \pi_{\Sigma}^{*}\left(L_{p}^{-1}\right)\right) \in K(\tilde{\mathcal{N}}) .
\end{aligned}
$$

Using standard properties of stable bundles it can be shown that $R^{0}$ vanishes. Thus $\mathbf{M}$ is a vector bundle of rank $2 g-1$. Its total Chern class is a complicated $^{3}$ polynomial of the universal classes. Since $\operatorname{rank}(\mathbf{M})=2 g-1$, the Chern class $c_{2 g+r}(\mathbf{M}) \in H^{4 g+2 r}(\tilde{\mathcal{N}})$ vanishes for $r \geq 0$. According to

\footnotetext{
${ }^{3}$ It was calculated by Zagier in [Zag].
} 
(2), the cohomology of $\tilde{\mathcal{N}}$ is the tensor product of $H^{*}(\mathcal{J})$ and $H^{*}(\mathcal{N})$. Thus if we write $\tau_{S}=\prod_{i \in S} \tau_{i} \in H^{|S|}(\mathcal{J})$ for $S \subset\{1 \ldots 2 g\}$ and

$$
c_{2 g+r}(\mathbf{M})=\sum_{S \subset\{1 \ldots 2 g\}} \zeta_{S}^{r} \otimes \tau_{S}
$$

in the Künneth decomposition of (2) then we get the vanishing of each $\zeta_{S}^{r}$. Thus for every $r \geq 0$ and $S \subset\{1 \ldots 2 g\}$ we get a relation

$$
\zeta_{S}^{r} \in \mathbb{Q}\left[\alpha, \beta, \psi_{i}\right]
$$

of degree $4 g+2 r-|S|$. The polynomials $\zeta_{S}^{r}$ are called the Mumford relations.

Mumford conjectured, and it was first proved by Kirwan in [Kir], that the Mumford relations constitute a complete set of relations of the cohomology ring of $\mathcal{N}$.

By now a complete description of the Mumford relations and the ring structure of $H^{*}(\mathcal{N})$ is available (see [Bar], [Ki,Ne], [Si,Ti] and [Zag] and also [Tha2] for an introduction to the topology of $\mathcal{N}$ ).

\subsection{The moduli space of rank 2 stable Higgs bundles $\mathcal{M}$}

We denote by $\tilde{\mathcal{M}}_{2 k-1}$ the coarse moduli space of rank 2 stable Higgs bundles ${ }^{4}$ with degree $2 k-1$ over $\Sigma$, which was constructed as $\mathcal{M}(2,2 k-1, K)$ in [Nit]. For a fixed $\Sigma$ they are all isomorphic to each other. We write $\tilde{\mathcal{M}}$ for $\tilde{\mathcal{M}}_{1}$. It is a smooth, non-projective, quasi-projective variety of dimension $8 g-6$.

The determinant gives a map $\operatorname{det}_{\mathcal{M}}: \tilde{\mathcal{M}} \rightarrow T_{\mathcal{J}}^{*}$, defined by $\operatorname{det}_{\mathcal{M}}(E, \Phi)=$ $\left(\Lambda^{2} E, \operatorname{tr}(\Phi)\right)$. For any $\mathcal{L} \in T_{\mathcal{J}}^{*}$ the fibre $\operatorname{det}_{\mathcal{M}}^{-1}(\mathcal{L})$ will be denoted by $\mathcal{M}_{\mathcal{L}}$. Just as in the stable vector bundle case any two fibres of $\operatorname{det}_{\mathcal{M}}$ are isomorphic. Usually we will write $\mathcal{M}$ for $\mathcal{M}_{\mathcal{L}}$, when the Abelian Higgs bundle $\mathcal{L}$ has zero Higgs field.

Our main concern in this paper is $\mathcal{M}$. It is a non-projective, smooth quasi-projective variety of dimension $6 g-6$. It was first introduced in [Hit1] and then an algebro-geometric approach was given in [Nit] and in [Sim].

Similarly to (1) we have a $G$-action on $\tilde{\mathcal{M}}$ and on $T_{\mathcal{J}}^{*}$ such that:

$$
\tilde{\mathcal{M}}=\left(\mathcal{M} \times T_{\mathcal{J}}^{*}\right) / G
$$

\footnotetext{
${ }^{4}$ For definitions see Section 4 .
} 
This on the level of cohomology gives

$$
H^{*}(\tilde{\mathcal{M}}) \cong\left(H^{*}(\mathcal{M})\right)^{G} \otimes H^{*}\left(T_{\mathcal{J}}^{*}\right) \cong\left(H^{*}(\mathcal{M})\right)^{G} \otimes H^{*}(\mathcal{J}) .
$$

In the case of $\mathcal{M}$ however we do not have the triviality of the action of $G$ on $H^{*}(\mathcal{M})$, but nevertheless the cohomology ring of $\tilde{\mathcal{M}}$ is determined by the ring $\left(H^{*}(\mathcal{M})\right)^{G}$.

There is quite little known about the ring $H^{*}(\mathcal{M})$. The Poincare polynomial of it is calculated in [Hit1]. From that calculation we can easily calculate the Poincaré polynomial of $\left(H^{*}(\mathcal{M})\right)^{G}$. Nothing else is known about $H^{*}(\mathcal{M})$. We will return to this problem in a forthcoming paper [Ha, Th].

In this paper we settle another problem concerning the topology of $\mathcal{M}$. We calculate all intersection numbers of $\mathcal{M}$. Because $\mathcal{M}$ is non-compact we have to work with compactly supported cohomology. Moreover there is no compactly supported cohomology below the middle dimension $6 g-6$. Thus the only interesting intersection numbers come from the intersection form on $H_{c p t}^{6 g-6}(\mathcal{M})$. This space is $g$ dimensional and generated by the compactly supported cohomology classes of the components of the nilpotent cone, which is the zero fibre of the Hitchin map or in other words the locus of stable Higgs bundles with nilpotent Higgs field (cf. Corollary 5.4 of [Hau1]). By considering the virtual Dirac bundle which is the analogue of the virtual Mumford bundle we will prove in the last section of this paper that the ordinary cohomology classes of the components of the nilpotent cone are trivial. This shows that the intersection form on $H_{c p t}^{6 g-6}(\mathcal{M})$ is trivial, which is equivalent to Theorem 1.1.

As a conclusion it can be said that the analogue of the Mumford relations for the moduli space of Higgs bundles is Theorem 1.1.

\section{Hypercohomology}

In this section we recall the notion of hypercohomology of a complex from $[\mathrm{Gr}, \mathrm{Ha}]$, and list some properties of it, which we will use later.

Definition 3.1 Let

$$
\mathcal{A}=\left(A_{0} \stackrel{d}{\longrightarrow} A_{1} \stackrel{d}{\longrightarrow} A_{2} \longrightarrow \ldots\right)
$$

be a complex of coherent sheaves $A_{i}$ over an algebraic variety $X$. For a covering $\underline{U}=\left\{U_{\alpha}\right\}$ of $X$ and each $A_{i}$ we get the Čech cochain complex with 
boundary operator $\delta$ :

$$
\left(C^{0}\left(\underline{U}, A_{i}\right) \stackrel{\delta}{\longrightarrow} C^{1}\left(\underline{U}, A_{i}\right) \stackrel{\delta}{\longrightarrow} \ldots\right) .
$$

Clearly d induces operators

$$
\left(C^{j}\left(\underline{U}, A_{i}\right) \stackrel{d}{\longrightarrow} C^{j}\left(\underline{U}, A_{j}\right)\right)
$$

satisfying $\delta^{2}=d^{2}=d \delta+\delta d=0$ : and hence gives rise to a double complex

$$
\left\{C^{p, q}=C^{p}\left(\underline{U}, A_{q}\right) ; \delta, d\right\}
$$

The hypercohomology of the complex $\mathcal{A}$ is given by the cohomology of the total complex of the double complex $C^{p, q}$ :

$$
\mathbb{H}^{*}(X, \mathcal{A})=\lim _{\underline{U}} H^{*}\left(C^{*}(\underline{U}), D\right) .
$$

Moreover if $\mathcal{A}$ is a complex over $X$ and $f: X \rightarrow Y$ is a projective morphism then for every non-negative integer $i$ define the sheaf $\mathbb{R}^{i} f_{*}(\mathcal{A})$ over $Y$ by

$$
\mathbb{R}^{i} f_{*}(\mathcal{A})(U)=\mathbb{H}^{i}\left(f^{-1}(U), \mathcal{A}\right) .
$$

Finally, define the pushforward of a complex to be:

$$
f_{!}(\mathcal{A})=\mathbb{R}^{0} f_{*}(\mathcal{A})-\mathbb{R}^{1} f_{*}(\mathcal{A})+\mathbb{R}^{2} f_{*}(\mathcal{A})-\ldots \in K(Y) .
$$

Remark. In this paper we will work only with two term complexes.

There is one important property of hypercohomology which we will make constant use of. If

$$
0 \rightarrow \mathcal{A} \rightarrow \stackrel{\mathcal{B}}{\rightarrow} \rightarrow \mathcal{C} \rightarrow 0
$$

is a short exact sequence of complexes then there is a long exact sequence of hypercohomology vector spaces:

$$
0 \rightarrow \mathbb{H}^{0}(X, \mathcal{A}) \rightarrow \mathbb{H}^{0}(X, \mathcal{B}) \rightarrow \mathbb{H}^{0}(X, \mathcal{C}) \rightarrow \mathbb{H}^{1}(X, \mathcal{A}) \rightarrow \ldots
$$


As an example consider the short exact sequence of two term complexes:

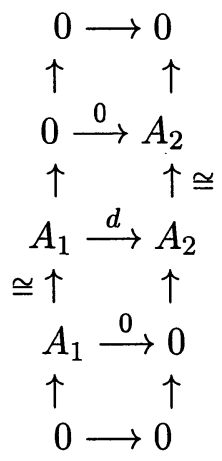

The long exact sequence in this case is:

$$
0 \rightarrow \mathbb{H}^{0}(X, \mathcal{A}) \rightarrow H^{0}\left(X, A_{1}\right) \rightarrow H^{0}\left(X, A_{2}\right) \rightarrow \mathbb{H}^{1}(X, \mathcal{A}) \rightarrow \ldots
$$

which we will call the hypercohomology long exact sequence of the two term complex $\mathcal{A}=A_{1} \stackrel{d}{\rightarrow} A_{2}$.

Consequently if $\mathcal{A}=A_{1} \stackrel{d}{\rightarrow} A_{2}$ is a two term complex over $X$ and $f$ : $X \rightarrow Y$ is a projective morphism then we have:

$$
0 \rightarrow \mathbb{R}^{0} f_{*}(X, \mathcal{A}) \rightarrow R^{0} f_{*}\left(X, A_{1}\right) \rightarrow R^{0} f_{*}\left(X, A_{2}\right) \rightarrow \mathbb{R}^{1} f_{*}(X, \mathcal{A}) \rightarrow \ldots,
$$

a long exact sequence of sheaves over $Y$.

\section{A vanishing theorem}

Definition 4.1 The complex $E \stackrel{\Phi}{\rightarrow} E \otimes K$ with $E$ a vector bundle on $\Sigma, K$ the canonical bundle of $\Sigma$, and $\Phi \in H^{0}(\Sigma, \operatorname{Hom}(E, E \otimes K))$, is called a Higgs bundle, while $\Phi$ is called the Higgs field.

We define a morphism $\Psi: \mathcal{E}_{1} \rightarrow \mathcal{E}_{2}$ between two Higgs bundles $\mathcal{E}_{1}=E_{1} \stackrel{\Phi_{1}}{\rightarrow} E_{1} \otimes K$ and $\mathcal{E}_{2}=E_{2} \stackrel{\Phi_{2}}{\rightarrow} E_{2} \otimes K$ to be a homomorphism of vector bundles $\Psi \in \operatorname{Hom}\left(E_{1}, E_{2}\right)$ such that the following diagram commutes:

$$
\begin{gathered}
E_{1} \stackrel{\Phi_{1}}{\longrightarrow} E_{1} \otimes K \\
\Psi \downarrow \\
E_{2} \stackrel{\Phi_{2}}{\longrightarrow} E_{2} \otimes K
\end{gathered}
$$


Moreover we say that $\mathcal{E}_{1}$ is a Higgs subbundle of $\mathcal{E}_{2}$ if $\Psi \in \operatorname{Hom}\left(E_{1}, E_{2}\right)$ is injective and a morphism of Higgs bundles. We denote this by $\mathcal{E}_{1} \subset \mathcal{E}_{2}$. In this case we can easily construct the quotient Higgs bundle $\mathcal{E}_{2} / \mathcal{E}_{1}$ together with a surjective morphism of Higgs bundles $\pi: \mathcal{E}_{2} \rightarrow \mathcal{E}_{2} / \mathcal{E}_{1}$ whose kernel is exactly $\mathcal{E}_{1}$.

Remark. It is a tautology that morphisms of Higgs bundles form the hypercohomology 5 vector space $\mathbb{H}^{0}\left(\Sigma, E_{1}^{*} \otimes E_{2} \stackrel{\left[\Phi_{1}, \Phi_{2}\right]}{\longrightarrow} E_{1}^{*} \otimes E_{2} \otimes K\right)$ where the homomorphism $\left[\Phi_{1}, \Phi_{2}\right]$ is given by: $\left[\Phi_{1}, \Phi_{2}\right](\Psi):=\left(\Psi \otimes i d_{K}\right) \Phi_{1}-\Phi_{2} \Psi$ for $\Psi \in \operatorname{Hom}\left(E_{1}, E_{2}\right)$.

Now we can define the notion of stability of Higgs bundles:

Definition 4.2 If $E$ is a vector bundle over $\Sigma$ then its slope is defined by $\mu(E):=\operatorname{deg}(E) / \operatorname{rank}(E)$. The slope $\mu(\mathcal{E})$ of a Higgs bundle $\mathcal{E}=E \stackrel{\Phi}{\rightarrow} E \otimes K$ is defined as the slope $\mu(E)$ of its vector bundle $E$. Now a Higgs bundle is called stable if it has strictly larger slope than any of its proper Higgs subbundles.

The main result of this section is the following theorem, the second part of which is Proposition (3.15) in [Hit1]:

Theorem 4.3 Let $\mathcal{E}=E \stackrel{\Phi}{\rightarrow} E \otimes K$ and $\mathcal{F}=F \stackrel{\Psi}{\rightarrow} F \otimes K$ be stable Higgs bundles with $\mu(\mathcal{F})<\mu(\mathcal{E})$. Then the only morphism from $\mathcal{E}$ to $\mathcal{F}$ is the trivial one. In other words

$$
\mathbb{H}^{0}\left(\Sigma, E^{*} \otimes F \stackrel{[\Phi, \Psi]}{\longrightarrow} E^{*} \otimes F \otimes K\right)=0 .
$$

Moreover if $\mu(\mathcal{F})=\mu(\mathcal{E})$, then there is a non-trivial morphism $f: \mathcal{E} \rightarrow \mathcal{F}$ if and only if $\mathcal{E} \cong \mathcal{F}$ in which case every non-trivial morphism $f: \mathcal{E} \rightarrow \mathcal{F}$ is an isomorphism and

$$
\operatorname{dim}\left(\mathbb{H}^{0}\left(\Sigma, E^{*} \otimes F \stackrel{[\Phi, \Psi]}{\longrightarrow} E^{*} \otimes F \otimes K\right)\right)=1 .
$$

\footnotetext{
${ }^{5}$ In connection with Higgs bundles the language of hypercohomology was first used in [Sim]. In $[\mathrm{Bi}, \mathrm{Ra}]$ it was used to describe the tangent space to $\mathcal{M}$.
} 
Proof. For the proof we need a lemma of Narasimhan and Seshadri (cf. section 4 in $[\mathrm{Na}, \mathrm{Se}])$ :

Lemma 4.4 Let $E$ and $F$ be two vector bundles over the Riemann surface $\Sigma$ with a non-zero homomorphism $f: E \rightarrow F$, then $f$ has the following canonical factorisation:

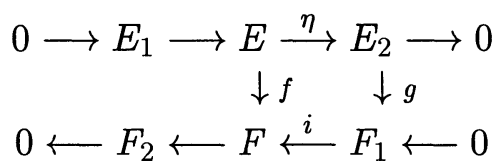

where $E_{1}, E_{2}, F_{1}$ and $F_{2}$ are vector bundles, each row is exact, $f=$ ig $\eta$ and $g$ is of maximal rank, i.e. $\operatorname{rank}\left(E_{2}\right)=\operatorname{rank}\left(F_{1}\right)=n$ and $\Lambda^{n}(g): \Lambda^{n}\left(E_{2}\right) \rightarrow$ $\Lambda^{n}\left(F_{1}\right)$ is a non-zero homomorphism. In other words $g$ is an isomorphism on a Zariski open subset $U$ of $\Sigma . F_{1}$ is called the subbundle of $F$ generated by the image of $f$.

Let $f: \mathcal{E} \rightarrow \mathcal{F}$ be a non-zero morphism of Higgs bundles. In particular $f: E \rightarrow F$ is a homomorphism of vector bundles.

Construct the canonical factorisation of $f$ of the above lemma. Consider the Zariski open subset $U$ of $\Sigma$ where $g$ is an isomorphism. Here clearly $\operatorname{ker}\left(\left.f\right|_{U}\right)=\operatorname{ker}\left(\left.\eta\right|_{U}\right)=\left.E_{1}\right|_{U}$. Now $\operatorname{ker}\left(\left.f\right|_{U}\right)$ being the kernel of a morphism of Higgs bundles is $\Phi$-invariant, i.e. a Higgs subbundle of $\left.\mathcal{E}\right|_{U}$. Thus $\left.E_{1}\right|_{U}$ is a Higgs subbundle of $\left.\mathcal{E}\right|_{U}$. This means that $\Phi\left(E_{1}\right)$ is contained in $E_{1} \otimes K \subset$ $E \otimes K$ on $U$. Because $U$ is Zariski open in $\Sigma$ it follows that $\mathcal{E}_{1}=E_{1} \stackrel{\Phi}{\rightarrow} E_{1} \otimes K$ is a Higgs subbundle of $\mathcal{E}$. Let $\mathcal{E}_{2}=E_{2} \stackrel{\tilde{\Phi}}{\rightarrow} E_{2} \otimes K$ denote the quotient Higgs bundle.

Similarly $\left.\operatorname{im}(\alpha)\right|_{U}=\left.F_{1}\right|_{U}$ is $\Psi$-invariant, thus $\mathcal{F}_{1}=F_{1} \stackrel{\Psi}{\rightarrow} F_{1} \otimes K$ is a Higgs subbundle of $F$.

By assumption $\mu(\mathcal{F})<\mu(\mathcal{E})$, stability of $\mathcal{E}$ gives $\mu(\mathcal{E}) \leq \mu\left(\mathcal{E}_{2}\right)$ (it may happen that $\left.E=E_{2}\right)$ and because $g$ is of maximal rank we get $\mu\left(\mathcal{E}_{2}\right)=$ $\mu\left(E_{2}\right) \leq \mu\left(F_{1}\right)=\mu\left(\mathcal{F}_{1}\right)$. Thus $\mu(\mathcal{F})<\mu\left(\mathcal{F}_{1}\right)$ contradicting the stability of $F$.

If $\mu(\mathcal{E})=\mu(\mathcal{F})$ then the above argument leaves the only possibility that $\eta, g$ and $i$ are isomorphisms, showing that $f$ must be an isomorphism. Suppose that we have such an isomorphism $f$ of Higgs bundles. Then consider $h: \mathcal{E} \rightarrow \mathcal{F}$ another non-zero morphism of Higgs bundles. In particular $h \in \operatorname{Hom}(E, F)$. Let $\lambda$ be an eigenvalue of the homomorphism 
$f_{p}^{-1} h_{p} \in \operatorname{Hom}\left(E_{p}, E_{p}\right)$. Then the homomorphism $h-\lambda f$ is not an isomorphism, though clearly a morphism of Higgs bundles. From the above argument this means that $h-\lambda f=0$.

The result follows.

Corollary 4.5 For any stable Higgs bundle $\mathcal{E}$ with $\mu(\mathcal{E})<0$ :

$$
\mathbb{H}^{0}(\Sigma, \mathcal{E})=0
$$

for any stable Higgs bundle $\mathcal{E}$ with $\mu(\mathcal{E})>0$ :

$$
\mathbb{H}^{2}(\Sigma, \mathcal{E})=0
$$

If $\mathcal{E}$ is a stable Higgs bundle with $\mu(\mathcal{E})=0$ and $\mathcal{E} \not \mathcal{E}_{0}=\mathcal{O}_{\Sigma} \stackrel{0}{\rightarrow} \mathcal{O}_{\Sigma} \otimes K$ then both (10) and (11) hold.

Proof. For the first part consider the Higgs bundle $\mathcal{E}_{0}=\mathcal{O}_{\Sigma} \stackrel{0}{\rightarrow} \mathcal{O}_{\Sigma} \otimes K$. Being of rank 1 it is obviously stable, with $\mu\left(\mathcal{E}_{0}\right)=0$. Now the previous theorem yields that there are no nontrivial morphisms from $\mathcal{E}_{0}$ to $\mathcal{E}$, which in the language of hypercohomology is exactly $\mathbb{H}^{0}(\Sigma, \mathcal{E})=0$, which we had to prove.

For the second part Serre duality gives that $\mathbb{H}^{2}(\Sigma, \mathcal{E}) \cong\left(\mathbb{H}^{0}\left(\Sigma, \mathcal{E}^{*} \otimes K\right)\right)^{*}$. Now clearly $\mathcal{E}^{*} \otimes K$ is stable and $\mu\left(\mathcal{E}^{*} \otimes K\right)=-\mu(\mathcal{E})<0$. Thus the first part gives the second.

Likewise, the third statement follows by referring to the last part of Theorem 4.3.

\section{Universal bundles}

Nitsure showed that $\tilde{\mathcal{M}}$ is a coarse moduli space. Here we show that $\tilde{\mathcal{M}}$ is in fact a fine moduli space. We closely follow the proof of Theorem 5.12 in [New] and (1.19) of [Tha3]. All the ingredients have already appeared in the unpublished [Tha1].

Definition 5.1 Two families $\mathcal{E}_{T}$ and $\mathcal{E}_{T}^{\prime}$ of stable Higgs bundles over $T \times \Sigma$ are said to be equivalent, (in symbols $\mathcal{E}_{T} \sim \mathcal{E}_{T}^{\prime}$ ) if there exists a line bundle $L$ on $T$ such that $\mathcal{E}_{T}^{\prime} \cong \mathcal{E}_{T} \otimes \pi_{T}^{*}(L)$. 
The next lemma, which is taken from [Tha1], shows that two families are equivalent iff they give rise to the same map to the coarse moduli space $\tilde{\mathcal{M}}$.

Lemma 5.2 If $\mathcal{E}_{T}=\mathbb{E}_{T} \stackrel{\Phi}{\rightarrow} \mathbb{E}_{T} \otimes K_{\Sigma}$ and $\mathcal{E}_{T}^{\prime}=\mathbb{E}_{T}^{\prime} \stackrel{\Phi^{\prime}}{\rightarrow} \mathbb{E}_{T}^{\prime} \otimes K_{\Sigma}$ are families of stable Higgs bundles over $T \times \Sigma$ such that

$$
\left.\left.\mathcal{E}_{T}\right|_{\{t\} \times \Sigma} \cong \mathcal{E}_{T}^{\prime}\right|_{\{t\} \times \Sigma}
$$

for each $t \in T$, then $\mathcal{E}_{T} \sim \mathcal{E}_{T}^{\prime}$.

Proof. Let $\mathcal{F}:=\mathbb{E}_{T}^{*} \otimes \mathbb{E}_{T}^{\prime} \stackrel{\left[\boldsymbol{\Phi}_{T}, \boldsymbol{\Phi}_{T}^{\prime}\right]}{\longrightarrow} \mathbb{E}_{T}^{*} \otimes \mathbb{E}_{T}^{\prime} \otimes K_{\Sigma}$. We define $L=\mathbb{R}^{0} \pi_{T *}(\mathcal{F})$. By (12) and (9) this is a line bundle over $T$. By the projection formula the sheaf $\mathbb{R}^{0} \pi_{T *}\left(\mathcal{F} \otimes \pi_{T}^{*}\left(L^{*}\right)\right)$ is just $\mathcal{O}_{T}$, the structure sheaf. A non-zero section $\Psi \in H^{0}\left(T, \mathbb{R}^{0} \pi_{T *}\left(\mathcal{F} \otimes \pi_{T}^{*}\left(L^{*}\right)\right)\right)$ for every $t \in T$ gives $\left.\Psi\right|_{\{t\} \times \Sigma}$ : $\left.\left.\left(\mathcal{E}_{T} \otimes \pi_{T}^{*}(L)\right)\right|_{\{t\} \times \Sigma} \rightarrow \mathcal{E}_{T}^{\prime}\right|_{\{t\} \times \Sigma}$ a non-zero morphism of Higgs bundles, which is by Theorem 4.3 an isomorphism.

The result follows.

Now we prove the existence of universal Higgs bundles (cf. [Tha1]):

Proposition 5.3 Universal Higgs bundles $\mathcal{E}_{\tilde{\mathcal{M}}}=\mathbb{E}_{\tilde{\mathcal{M}}} \stackrel{\Phi}{\rightarrow} \mathbb{E}_{\tilde{\mathcal{M}}} \otimes K_{\Sigma}$ over $\tilde{\mathcal{M}} \times \Sigma$ do exist.

Proof. The proof is analogous to the proof of Theorem 5.12 of [New] using the GIT construction of Nitsure [Nit] (cf. also (1.19) of [Tha3]).

First we recall the construction of $\tilde{\mathcal{M}}_{2 k-1}$ from [Nit]. Let $n=2 k-1+$ $2(1-g)$ with $k$ large enough. Then by Corollary 3.4 of [Nit] for any stable Higgs bundle $E \stackrel{\Phi}{\rightarrow} E \otimes K, E$ is a quotient of $\mathcal{O}_{\Sigma}^{n}$. Let $\mathcal{Q}$ be the quot scheme of all quotient sheaves $\mathcal{O}_{\Sigma}^{N} \rightarrow \mathcal{F}$ of rank 2 and degree $d$. Let $\mathcal{O}_{\Sigma \times \mathcal{Q}}^{n} \rightarrow U$ be the universal quotient sheaf on $\Sigma \times \mathcal{Q}$. Let $R \subset \mathcal{Q}$ be the subset of all $q$ for which $\mathcal{F}_{q}$ is locally free and the map $H^{0}\left(\Sigma, \mathcal{O}_{\Sigma}^{n}\right) \rightarrow H^{0}\left(\Sigma, U_{q}\right)$ is an isomorphism.

It follows from Proposition 3.6 of [Nit] that there exists a locally universal family for stable Higgs bundles of degree 2 and degree $2 k-1$ given by $\mathcal{E}_{s}=\mathbb{E}_{s} \stackrel{\Phi_{s}}{\longrightarrow} \mathbb{E}_{s} \otimes K_{\Sigma}$ over $F_{s} \times \Sigma$ where $F_{s}$ is an open subset of a linear $R$-scheme $F \rightarrow R$ and $\mathcal{E}_{s}=\left.\mathcal{E}_{F}\right|_{F_{s} \times \Sigma}$ where $\mathcal{E}_{F}=\mathbb{E}_{F} \stackrel{\boldsymbol{\Phi}_{F}}{\longrightarrow} \mathbb{E}_{F} \otimes K_{\Sigma}$ is a family of Higgs bundles over $F$. 
First by Theorem 5.3 of [New] $G L(n)$ acts on $R$. Now $G L(n)$ acts equivariantly on the $R$-scheme $F \rightarrow R$, which gives a $G L(n)$ equivariant complex $\mathcal{E}_{F}$. The centre of $G L(n)$ acts trivially on $F$ and by multiplication on $\mathcal{E}_{F}$. Nitsure constructs $\tilde{\mathcal{M}}_{2 k-1}$ in Theorem 5.10 of [Nit] as a good quotient of $F_{s}$ by $P G L(n) \cong G L(n) / Z(G L(n))$.

The proof of Lemma 5.11 of [New] gives a $G L(n)$-equivariant line bundle $L$ over $R$ (although in Lemma 5.11 of [New] $L$ is constructed only over $R_{s}$ the same construction works over the whole $R$ ) for which $Z(G L(n))$ acts on $L$ by scalar multiplication. Now for the $G L(n)$-equivariant bundle $\mathcal{E}_{F} \otimes\left(\pi_{F}\right.$ 。 $g)^{*}\left(L^{-1}\right)$ the centre acts trivially thus it descends to a $P G L(n)$-equivariant complex over $F \times \Sigma$. This gives a $P G L(n)$-equivariant locally universal family $\mathcal{E}_{s} \otimes\left(\pi_{F} \circ g\right)^{*}\left(L^{-1}\right)$ over $F_{s} \times \Sigma$. By Kempf's descent lemma (cf. Theorem 2.3 of $[\mathrm{Dr}, \mathrm{Na}]))$ the $P G L(n)$-equivariant bundle $\mathbb{E}_{s} \times\left(\pi_{F} \circ g\right)^{*}\left(L^{-1}\right)$ descends to a bundle to the good quotient $\tilde{\mathcal{M}}_{2 k-1} \times \Sigma$ and since the section $\Phi_{s}$ is invariant, it also descends. Clearly the resulting complex $\mathcal{E}_{\tilde{\mathcal{M}}_{2 k-1}}$ then will be a universal Higgs bundle over $\tilde{\mathcal{M}}_{2 k-1}$. (A similar situation appears in (1.19) of [Tha3].)

Finally from a universal Higgs bundle over $\tilde{\mathcal{M}}_{2 k-1}$ one can easily construct universal Higgs bundles over any $\tilde{\mathcal{M}}_{2 l-1}$.

The result follows.

As in Theorem 5.12 of [New] and (1.19) of [Tha3] our Lemma 5.2 and Proposition 5.3 gives:

Corollary 5.4 The space $\tilde{\mathcal{M}}$ is a fine moduli space for rank 2 stable Higgs bundles of degree 1 with respect to the equivalence $\sim$ of families of stable Higgs bundles.

As another consequence of Proposition 5.3 and Lemma 5.2 we see that although $\mathbb{E}_{\tilde{\mathcal{M}}}$ is not unique $\operatorname{End}\left(\mathbb{E}_{\tilde{\mathcal{M}}}\right)$ is. Moreover it is clear that by setting $\mathbb{E}_{\mathcal{M}}=\left.\mathbb{E}_{\tilde{\mathcal{M}}}\right|_{\mathcal{M} \times \Sigma}$ we have

$$
c\left(\operatorname{End}\left(\mathbb{E}_{\tilde{\mathcal{M}}}\right)\right)=c\left(\operatorname{End}\left(\mathbb{E}_{\mathcal{M}}\right)\right) \otimes 1
$$

in the decomposition (5).

Thus from the Künneth decomposition of $\operatorname{End}\left(\mathbb{E}_{\mathcal{M}}\right)$ we get universal classes

$$
c_{2}\left(\operatorname{End}\left(\mathbb{E}_{\mathcal{M}}\right)\right)=2 \alpha_{\mathcal{M}} \otimes \sigma+\sum_{i=1}^{2 g} 4 \psi_{\mathcal{M}}^{i} \otimes e_{i}-\beta_{\mathcal{M}} \otimes 1
$$


in $H^{4}(\mathcal{M} \times \Sigma) \cong \sum_{r=0}^{4} H^{r}(\mathcal{M}) \otimes H^{4-r}(\Sigma)$ for some $\alpha_{\mathcal{M}} \in H^{2}(\mathcal{M}), \psi_{\mathcal{M}}^{i} \in$ $H^{3}(\mathcal{M})$ and $\beta_{\mathcal{M}} \in H^{4}(\mathcal{M})$.

Clearly $\left.\alpha_{\mathcal{M}}\right|_{\mathcal{N}}=\alpha_{\mathcal{N}},\left.\psi_{\mathcal{M}}^{i}\right|_{\mathcal{N}}=\psi_{\mathcal{N}}^{i}$ and $\left.\beta_{\mathcal{M}}\right|_{\mathcal{N}}=\beta_{\mathcal{N}}$.

Though $\mathbb{E}_{\mathcal{M}}$ is not unique we can still write its Chern classes in the Künneth decomposition (cf. proof of Newstead's theorem in [Tha2]), getting $c_{1}\left(\mathbb{E}_{\mathcal{M}}\right)=1 \otimes \sigma+\beta_{1} \otimes 1$, where $\beta_{1} \in H^{2}(\mathcal{M})$ (note that $\mathcal{M}$ being simply connected by [Hit1] $\left.H^{1}(\mathcal{M})=0\right)$ and $c_{2}\left(\mathbb{E}_{\mathcal{M}}\right)=\alpha_{2} \otimes \sigma+\sum_{i=1}^{2 g} a_{i} \otimes e_{i}+\beta_{2} \otimes 1$, where $\alpha_{2} \in H^{2}(\mathcal{M}), a_{i} \in H^{3}(\mathcal{M})$ and $\beta_{2} \in H^{4}(\mathcal{M})$. Because $4 c_{2}\left(\mathbb{E}_{\mathcal{M}}\right)-$ $c_{1}^{2}\left(\mathbb{E}_{\mathcal{M}}\right)=c_{2}\left(\operatorname{End}\left(\mathbb{E}_{\mathcal{M}}\right)\right)$, we get $\alpha_{\mathcal{M}}=2 \alpha_{2}-\beta_{1}$ and $\beta=\beta_{1}^{2}-4 \beta_{2}$. Because $\operatorname{Pic}(\mathcal{M}) \cong H^{2}(\mathcal{M}, \mathbb{Z})$ (cf. [Hau1]) we can normalize $\mathbb{E}_{\mathcal{M}}$ uniquely such that $\beta_{1}=\alpha_{\mathcal{M}}$ i.e. $c_{1}\left(\mathbb{E}_{\mathcal{M}}\right)=1 \otimes \sigma+\alpha_{\mathcal{M}} \otimes 1$.

Definition 5.5 The universal Higgs bundle $\mathcal{E}_{\mathcal{M}}$ is normalized if $c_{1}\left(\left(\mathbb{E}_{\mathcal{M}}\right)_{p}\right)=\alpha_{\mathcal{M}}$, where $\left(\mathbb{E}_{\mathcal{M}}\right)_{p}:=\left.\mathbb{E}_{\mathcal{M}}\right|_{\mathcal{M} \times\{p\}}$.

We also need to work out the Chern classes of $\mathbb{E}_{\tilde{\mathcal{M}}}$. It is easy to see that $c\left(\mathbb{E}_{\tilde{\mathcal{M}}}\right)$ in the decomposition (5) is the product of $\left.c\left(\mathbb{E}_{\mathcal{\mathcal { M }}}\right)\right|_{\mathcal{M} \times \Sigma}$ and $c\left(\mathbb{L}_{1}\right)$, where $\mathbb{L}_{1}$ is some universal line bundle over $\mathcal{J} \times \Sigma$.

Definition 5.6 We call the universal Higgs bundle $\mathcal{E}_{\tilde{\mathcal{M}}}$ normalized if in the decomposition (5)

$$
c_{1}\left(\left(\mathbb{E}_{\tilde{\mathcal{M}}}\right)_{p}\right)=\alpha_{\mathcal{M}}
$$

where $\left(\mathbb{E}_{\tilde{\mathcal{M}}}\right)_{p}=\left.\mathbb{E}_{\tilde{\mathcal{M}}}\right|_{\tilde{\mathcal{M}} \times\{p\}}$.

Remark. Since $4 c_{2}\left(\left(\mathbb{E}_{\tilde{\mathcal{M}}}\right)_{p}\right)-c_{1}\left(\left(\mathbb{E}_{\tilde{\mathcal{M}}}\right)_{p}\right)^{2}=c_{2}\left(\operatorname{End}\left(\left(\mathbb{E}_{\tilde{\mathcal{M}}}\right)_{p}\right)\right)$, for a normalized universal Higgs bundle over $\tilde{\mathcal{M}} \times \Sigma(13)$ and (14) yield:

$$
c_{2}\left(\left(\mathbb{E}_{\tilde{\mathcal{M}}}\right)_{p}\right)=\frac{\left(\alpha_{\mathcal{M}}^{2}-\beta_{\mathcal{M}}\right)}{4}
$$

Finally, given a universal Higgs bundle $\mathcal{E}_{\tilde{\mathcal{M}}}$ over $\tilde{\mathcal{M}} \times \Sigma$, we introduce a universal Higgs bundle of degree $2 k-1$ by setting

$$
\mathcal{E}_{\tilde{\mathcal{M}}}^{k}:=\mathcal{E}_{\tilde{\mathcal{M}}} \otimes \pi_{\Sigma}^{*}\left(L_{p}^{k-1}\right)
$$

where $L_{p}$ is the line bundle of the divisor of the point $p \in \Sigma$. It is called normalized if $\mathcal{E}_{\tilde{\mathcal{M}}}$ is normalized. As a matter of fact $\mathcal{E}_{\tilde{\mathcal{M}}}^{k}$ can be thought of as a pull back of a universal Higgs bundle from $\tilde{\mathcal{M}}_{2 k-1} \times \Sigma$. 


\section{The virtual Dirac bundle, $\mathbf{D}_{k}$}

The strategy of the proof of Theorem 1.1 will be to examine the virtual Dirac bundle $\mathbf{D}_{k}$ which is defined in the following:

Definition 6.1 The virtual Dirac bundle $i s^{6}$

$$
\mathbf{D}_{k}:=-\pi_{\tilde{\mathcal{M}} !}\left(\mathcal{E}_{\tilde{\mathcal{M}}}^{k}\right) \in K(\tilde{\mathcal{M}})
$$

where $\mathcal{E}_{\tilde{\mathcal{M}}}^{k}$ is a normalized universal Higgs bundle of degree $2 k-1$ and $\pi_{\tilde{\mathcal{M}}}$ : $\tilde{\mathcal{M}} \times \Sigma \rightarrow \tilde{\mathcal{M}}$ is the projection to $\tilde{\mathcal{M}}$.

The name is justified by Hitchin's construction [Hit2] $]^{7}$ of $\mathbf{D}_{k}$ related to the space of solutions of an equation on $\Sigma$, which is locally the dimensional reduction of the Dirac equation in $\mathbb{R}^{4}$ coupled to a self-dual Yang-Mills field.

The virtual Dirac bundle is a priori

$$
\pi_{\mathcal{M} !}\left(\mathcal{E}_{\tilde{\mathcal{M}}}^{k}\right)=-\mathbb{R}^{0} \pi_{\tilde{\mathcal{M}} *}\left(\mathcal{E}_{\tilde{\mathcal{M}}}^{k}\right)+\mathbb{R}^{1} \pi_{\tilde{\mathcal{M}} *}\left(\mathcal{E}_{\tilde{\mathcal{M}}}^{k}\right)-\mathbb{R}^{2} \pi_{\tilde{\mathcal{M}} *}\left(\mathcal{E}_{\tilde{\mathcal{M}}}^{k}\right)
$$

a formal sum of three coherent sheaves. Corollary 4.5 shows that one of these sheaves always vanishes: if $k>0$, then $\mathbb{R}^{2}=0$, if $k \leq 0$ then $\mathbb{R}^{0}=0$. From now on $k$ is assumed to be positive.

In this section we show that we can think of the virtual Dirac bundle as the virtual degeneracy sheaf of a homomorphism of vector bundles. More precisely we prove:

Theorem 6.2 There exist two vector bundles $V$ and $W$ over $\tilde{\mathcal{M}}$ together with a homomorphism $f: V \rightarrow W$ of vector bundles, whose kernel and cokernel are respectively $\mathbb{R}^{0} \pi_{\mathcal{M} *}\left(\mathcal{E}_{\tilde{\mathcal{M}}}^{k}\right)$ and $\mathbb{R}^{1} \pi_{\mathcal{M} *}\left(\mathcal{E}_{\tilde{\mathcal{M}}}^{k}\right)$. In other words there is an exact sequence of sheaves:

$$
0 \rightarrow \mathbb{R}^{0} \pi_{\tilde{\mathcal{M}} *}\left(\mathcal{E}_{\tilde{\mathcal{M}}}^{k}\right) \rightarrow V \stackrel{f}{\rightarrow} W \rightarrow \mathbb{R}^{1} \pi_{\tilde{\mathcal{M}} *}\left(\mathcal{E}_{\tilde{\mathcal{M}}}^{k}\right) \rightarrow 0 .
$$

Proof $^{\beta}$. First we need a lemma.

\footnotetext{
${ }^{6}$ Recall the definition of the pushforward of a complex from Section 3.

${ }^{7}$ Cf. Subsection 1.1.5 of [Hau2].

${ }^{8}$ The idea of the proof was suggested by Manfred Lehn.
} 
Lemma 6.3 Let $X$ be a smooth quasi-projective variety and $\Sigma$ a smooth projective curve. If $E$ is a locally free sheaf over $X \times \Sigma$ then there exists a vector bundle $F$ over $X \times \Sigma$ with a surjective vector bundle homomorphism $g_{E}: F \rightarrow E$ such that $R^{0} \pi_{X *}(F)=0$. We will call $F$ a sectionless resolution of $E$.

Proof. The lemma is a special case of Proposition 2.1.10 of [Hu,Le]. We have to only note that $X$ as an algebraic variety is a $\mathbb{C}$-scheme of finite type, $\pi_{X *}: X \times \Sigma \rightarrow X$ is clearly a smooth projective morphism of relative dimension 1 and $E$ being locally free is flat over $X$.

The proof is rather simple so we sketch it here. Let us denote by $E_{x}$ the vector bundle $\left.E\right|_{\{x\} \times \Sigma}$ over $\Sigma$. Fix an ample line bundle $L$ on $\Sigma$. Then it is well known that for big enough $k$ the vector bundle $E_{x} \otimes L^{k}$ is generated by its sections and $H^{1}\left(\Sigma ; E_{x} \otimes L^{k}\right)=0$. Let us denote by $X_{k} \subset X$ those points $x$ for which $E_{x} \otimes L^{k}$ is generated by its sections and $H^{1}\left(\Sigma ; E_{x} \otimes L^{k}\right)=0$. It is standard that $X_{k}$ is a Zariski open subset of $X$. Thus we have a covering $X=\bigcup X_{k}$ of $X$ by Zariski open subsets. It is again standard that the Zariski topology of an algebraic variety is noetherian ${ }^{9}$, which yields that we have some $k$ such that $X_{k}=X$. It is now immediate that

$$
F=\pi_{\Sigma}^{*}\left(L^{-k}\right) \otimes \pi_{X}^{*}\left(\left(\pi_{X}\right)_{*}\left(E \otimes \pi_{\Sigma}^{*}\left(L^{k}\right)\right)\right)
$$

has the required properties.

The result follows.

Proposition 6.4 Let $\Sigma$ be a smooth projective curve and $X$ be a smooth quasi-projective variety. Let $\mathcal{E}=E \stackrel{f}{\rightarrow} F$ be a complex of vector bundles on $X \times \Sigma$. Let $g_{F}: A \rightarrow F$ be a sectionless resolution of $F$. Let $M$ be the fibred product of $f$ and $g_{F}$. This comes with projection maps $p_{F}: M \rightarrow F$ and $p_{A}: M \rightarrow A$. Let $g_{M}: A_{2} \rightarrow M$ be a sectionless resolution of $M$, and denote $j=g_{M} \circ p_{A_{2}}$. Finally, let $A_{1}=\operatorname{ker} g_{M}$ and $i: A_{1} \rightarrow A_{2}$ the embedding. The situation is shown in the following diagram:

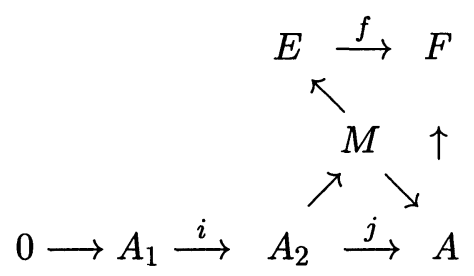

${ }^{9}$ Cf. Example 3.2 .1 on p. 84 of [Har]. 
In this case the cohomology of the complex

$$
R^{1} \pi_{X *}\left(A_{1}\right) \stackrel{i_{*}}{\longrightarrow} R^{1} \pi_{X *}\left(A_{2}\right) \stackrel{j_{*}}{\longrightarrow} R^{1} \pi_{X *}(A)
$$

calculates the sheaves $\mathbb{R}^{0} \pi_{X *}(\mathcal{E}), \mathbb{R}^{1} \pi_{X *}(\mathcal{E})$ and $\mathbb{R}^{2} \pi_{X *}(\mathcal{E})$ respectively. In other words

$$
\begin{aligned}
& \mathbb{R}^{0} \pi_{X *}(\mathcal{E}) \cong \operatorname{ker}\left(i_{*}\right) \\
& \mathbb{R}^{1} \pi_{X *}(\mathcal{E}) \cong \operatorname{ker}\left(j_{*}\right) / \operatorname{im}\left(i_{*}\right) \\
& \mathbb{R}^{2} \pi_{X *}(\mathcal{E}) \cong \operatorname{coker}\left(j_{*}\right)
\end{aligned}
$$

Proof. Let us recall the definition of the fibred product: $M:=\operatorname{ker}\left(f-g_{F}\right.$ : $E \oplus A \rightarrow F)$. This comes equipped with two obvious projections $p_{E}: M \rightarrow E$ and $p_{A}: M \rightarrow A$. Because $g_{F}$ is surjective $f-g_{F}$ is also surjective. Thus $M$ is a vector bundle. By construction the kernel of $p_{E}$ is isomorphic to the kernel of $g_{F}$. Denote it by $B$. This says that the following diagram is commutative and has two exact columns:

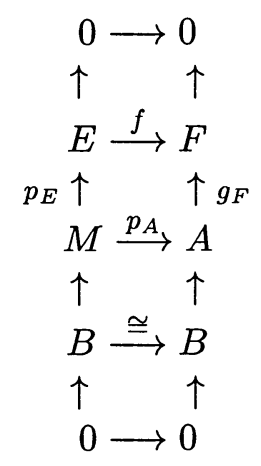

If $\mathcal{A}$ denotes the complex $\mathcal{A}=M \stackrel{p_{A}}{\rightarrow} A$ and $\mathcal{B}$ the complex $\mathcal{B}=B \stackrel{\cong}{\rightarrow} B$, then the above diagram is just a short exact sequence of complexes

$$
0 \longrightarrow \mathcal{B} \longrightarrow \mathcal{A} \longrightarrow \mathcal{E} \longrightarrow 0 .
$$

Clearly $\mathbb{R}^{i} \pi_{X *}(\mathcal{B})$ vanishes for all $i$. (Any hypercohomology of an isomorphism is 0 .) Thus the long exact sequence of the above short exact sequence gives the isomorphisms

$$
\begin{aligned}
& \mathbb{R}^{0} \pi_{X *}(\mathcal{E}) \cong \mathbb{R}^{0} \pi_{X *}(\mathcal{A}) \\
& \mathbb{R}^{1} \pi_{X *}(\mathcal{E}) \cong \mathbb{R}^{1} \pi_{X *}(\mathcal{A}) \\
& \mathbb{R}^{2} \pi_{X *}(\mathcal{E}) \cong \mathbb{R}^{2} \pi_{X *}(\mathcal{A})
\end{aligned}
$$


Because $A$ is a sectionless resolution of $M$, we have $R^{0} \pi_{X *}(A)=0$ thus the long exact sequence of the push forward of the complex $\mathcal{A}$ breaks up into two exact sequences:

$$
0 \rightarrow \mathbb{R}^{0} \pi_{X *}(\mathcal{A}) \rightarrow R^{0} \pi_{X *}(M) \rightarrow 0
$$

and

$$
0 \longrightarrow \mathbb{R}^{1} \pi_{X *}(\mathcal{A}) \longrightarrow R^{1} \pi_{\mathcal{W}_{*}}(M) \stackrel{p_{A_{*}}}{\longrightarrow} R^{1} \pi_{X *}(A) \longrightarrow \mathbb{R}^{2} \pi_{X *}(\mathcal{A}) \longrightarrow 0 .
$$

Thus

$$
\begin{aligned}
& \mathbb{R}^{0} \pi_{X *}(\mathcal{A}) \cong R^{0} \pi_{X_{*}}(M) \\
& \mathbb{R}^{1} \pi_{X_{*}}(\mathcal{A}) \cong \operatorname{ker}\left(p_{A_{*}}\right) \\
& \mathbb{R}^{2} \pi_{X_{*}}(\mathcal{A}) \cong \operatorname{coker}\left(p_{A_{*}}\right)
\end{aligned}
$$

Now consider the short exact sequence:

$$
0 \longrightarrow A_{1} \stackrel{i}{\longrightarrow} A_{2} \stackrel{g_{M}}{\longrightarrow} M \longrightarrow 0 \text {. }
$$

$R^{0} \pi_{X *}\left(A_{2}\right)=0$ because $A_{2}$ is a sectionless resolution of $M$ and hence we get the exact sequence of sheaves:

$$
\begin{aligned}
0 & \longrightarrow R^{0} \pi_{X *}(M) \longrightarrow R^{1} \pi_{X *}\left(A_{1}\right) \stackrel{i_{*}}{\longrightarrow} R^{1} \pi_{X *}\left(A_{2}\right)^{g_{M *}} \\
& \longrightarrow R^{1} \pi_{\mathcal{W}_{*}}(M) \longrightarrow 0
\end{aligned}
$$

Thus $\operatorname{ker}\left(i_{*}\right) \cong R^{0} \pi_{X *}(M)$ which by $(22)$ and (19) proves (16).

Since $g_{M_{*}}$ is a surjection $\operatorname{coker}\left(j_{*}\right) \cong \operatorname{coker}\left(p_{A_{*}}\right)$. This together with (24) and (21) gives (18).

Finally, consider the commutative diagram:

$$
\begin{array}{cc}
R^{1} \pi_{\mathcal{W}_{*}}(M) \stackrel{\cong}{\longrightarrow} R^{1} \pi_{X *}(M) \\
g_{M_{*}} \uparrow & \downarrow p_{A_{*}} \\
R^{1} \pi_{X *}\left(A_{2}\right) \stackrel{j_{*}}{\longrightarrow} R^{1} \pi_{X *}(A)
\end{array}
$$

Since $g_{M_{*}}$ surjective by (25) we get that $\operatorname{ker}\left(j_{*}\right) / \operatorname{ker}\left(g_{M_{*}}\right) \cong \operatorname{ker}\left(p_{A_{*}}\right)$. From (25) clearly $\operatorname{ker}\left(g_{M_{*}}\right) \cong \operatorname{im}\left(i_{*}\right)$, thus $\operatorname{ker}\left(j_{*}\right) / \operatorname{im}\left(i_{*}\right) \cong \operatorname{ker}\left(p_{A_{*}}\right)$. This together with (23) and (20) proves (17).

Corollary 6.5 If $\mathbb{R}^{2} \pi_{X *}(\mathcal{E})=0$, in the situation of Proposition 6.4, then there exist two vector bundles $V$ and $W$ over $X$ together with a homomorphism $f: V \rightarrow W$, whose kernel and cokernel are $\mathbb{R}^{0} \pi_{X *}(\mathcal{E})$ and $\mathbb{R}^{1} \pi_{X *}(\mathcal{E})$ respectively. I.e. the following sequence is exact:

$$
0 \rightarrow \mathbb{R}^{0} \pi_{X *}(\mathcal{E}) \rightarrow V \stackrel{f}{\rightarrow} W \rightarrow \mathbb{R}^{1} \pi_{X *}(\mathcal{E}) \rightarrow 0
$$


Proof. From the long exact sequence corresponding to (6), we have $R^{0} \pi_{X *}\left(A_{1}\right)=0$. Let $V$ be the vector bundle $R^{1} \pi_{X *}\left(A_{1}\right)$.

Moreover $R^{1} \pi_{X *}\left(A_{2}\right)$ and $R^{1} \pi_{X *}(A)$ are also vector bundles because $A_{2}$ and $A$ are sectionless resolutions. Furthermore the assumption $\mathbb{R}^{2} \pi_{X *}(\mathcal{E})=$ 0 shows that $j_{*}$ is surjective. Let $W$ be the vector bundle $\operatorname{ker}\left(j_{*}\right)$, and $f$ be the map $i_{*}: V \rightarrow W$.

The result follows from Proposition 6.4.

The proof of Theorem 6.2 is completed by Corollary 6.5 noting that by Corollary 4.5 we have $\mathbb{R}^{2} \pi_{\tilde{\mathcal{M}}_{*}}\left(\mathcal{E}_{\tilde{\mathcal{M}}}^{k}\right)=0$.

\section{The degeneracy locus $D_{k}$}

Definition 7.1 The degeneracy locus $\left.D_{k}:=\left\{\mathcal{E} \in \tilde{\mathcal{M}}: \mathbb{H}^{0}\left(\Sigma, \mathcal{E}_{\tilde{\mathcal{M}}}^{k}\right) \neq 0\right)\right\}$ is the locus ${ }^{10}$ where $\mathbf{D}_{k}$ fails to be a vector bundle, i.e. where $f$ of Theorem 6.2 fails to be an injection.

The aim of this section is to give a description of the degeneracy locus $D_{k}$. For this we need a refinement of Theorem 5.5 of [Hau1], which still follows from the proof of Proposition (19) of [Tha1].

Definition 7.2 The nilpotent cone $N \subset \mathcal{M}$ is the set of stable Higgs bundles with nilpotent Higgs field. In other words it is $\chi^{-1}(0)$ : the zero fibre of the Hitchin map.

Similarly $\tilde{N}:=\tilde{\chi}^{-1}(0) \subset \tilde{\mathcal{M}}$.

Proposition 7.3 The nilpotent cone is a compact union of $3 g-3$ dimensional manifolds:

$$
N=\mathcal{N} \cup \bigcup_{k=1}^{g-1} E_{k},
$$

where each $E_{k}$ is biholomorphic to the total space of a vector bundle over $N_{k}$, the $k$-th component of the fixed point set of the $\mathbb{C}^{*}$-action.

\footnotetext{
${ }^{10}$ For a rigorous construction of degeneracy loci cf. [ACGH] p.83. Our degeneracy locus is the $k$-th degeneracy locus of [ACGH], where $k=\operatorname{rank}(V)-1$.
} 
Moreover $E_{k}$ can be characterised as the locus of those stable Higgs bundles $\mathcal{E}=E \stackrel{\Phi}{\rightarrow} E \otimes K$ which have a unique subbundle $L_{\mathcal{E}}$ of degree $1-k$ killed by the non-zero Higgs field $\Phi$.

Proof. The first part is proved in Theorem 5.5 of [Hau1].

For the second part consider a universal Higgs bundle $\mathcal{E}_{\mathcal{M}}$ over $\mathcal{M} \times \Sigma$ restricted to $E_{k} \times \Sigma$. Let us denote it by $\mathcal{E}_{k}=\mathbb{E}_{k} \stackrel{\Phi_{k}}{\rightarrow} \mathbb{E}_{k} \times K_{\Sigma}$. Consider the kernel of $\boldsymbol{\Phi}_{k}$. Because $E_{k}$ parameterizes nilpotent stable Higgs bundles with non-zero Higgs field $\operatorname{ker}\left(\boldsymbol{\Phi}_{k}\right)$ is a line bundle over $E_{k} \times \Sigma$. Recall from Proposition 7.1 of [Hit1] that for $E \stackrel{\Phi}{\rightarrow} E \otimes K \in N_{k} \subset E_{k}$ we have $\operatorname{deg}(\operatorname{ker}(\Phi))=1-k$. Since $E_{k}$ is smooth we have that $\operatorname{deg}(\operatorname{ker}(\Phi))=1-k$ for every $E \stackrel{\Phi}{\rightarrow} E \otimes K \in E_{k}$.

The result follows.

Remark. Clearly a completely analogous result holds for $\tilde{N}$ with $\tilde{\mathcal{N}}, \tilde{E}_{k}$ and $\tilde{N}_{k}$ instead of $\mathcal{N}, E_{k}$ and $N_{k}$.

Notation 7.4 If $X$ is an irreducible locally closed subvariety of a smooth algebraic variety $Y$ of codimension $d$, then $\eta_{X}^{Y} \in H^{2 d}(Y)$ denotes the cohomology class of $\bar{X}$ in $Y$.

If $X$ is an irreducible locally closed and relatively complete subvariety of $Y$ then $\bar{\eta}_{X}^{Y} \in H_{\text {cpt }}^{2 d}(Y)$ denotes the compactly supported cohomology class of $\bar{X}$ in $Y$.

Theorem 7.5 Let $k=1, . ., g-1$. The degeneracy locus $D_{k}$ has the following decomposition:

$$
D_{k}=\tilde{\mathcal{N}}_{k} \cup \bigcup_{i=1}^{k} \tilde{E}_{i}^{k},
$$

where $\tilde{\mathcal{N}}_{k}=D_{k} \cap \tilde{\mathcal{N}}$, and $\tilde{E}_{i}^{k} \subset \tilde{E}_{i}$ are those nilpotent stable Higgs bundles whose unique line bundle $L_{\mathcal{E}}$ of Proposition 7.3 has the property that $H^{0}\left(\Sigma, L_{\mathcal{E}} \otimes L_{p}^{k-1}\right) \neq 0$.

Furthermore $\tilde{E}_{k}^{k}:=\left\{\mathcal{E} \in \tilde{E}_{k}: L_{\mathcal{E}}=L_{p}^{1-k}\right\}$ and hence

$$
\eta_{\tilde{E}_{k}^{k}}^{\tilde{\tilde{N}}} \backslash[\mathcal{J}]=\eta_{E_{k}}^{\mathcal{M}} \in H^{6 g-6}(\mathcal{M})
$$

where $\eta_{\widetilde{E}_{k}^{k}}^{\tilde{\mathcal{U}}} \backslash[\mathcal{J}]$ means the coefficient of $\eta_{p t}^{\mathcal{J}}$ in the decomposition of (5). 
Proof. Let $\mathcal{E}=E \stackrel{\Phi}{\rightarrow} E \otimes K$ be a stable Higgs bundle with $\Phi \neq 0$ and $\mathbb{H}^{0}\left(\Sigma, \mathcal{E} \otimes L_{p}^{k-1}\right) \neq 0$. It is easy to see that this hypercohomology is the vector space of all morphisms from $\mathcal{E}_{0} \otimes L_{p}^{1-k}=L_{p}^{1-k} \stackrel{0}{\rightarrow} L_{p}^{1-k} \otimes K$ to $\mathcal{E}$. Consider a nonzero such morphism $f$. Consider $L$ the line subbundle of $E$ generated by the image of $f$ of Lemma 4.4. Clearly $L$ is killed by the Higgs field $\Phi$. This shows that $\mathcal{E} \in \tilde{N}$ and $L=L_{\mathcal{E}}$. We also see that $\mathbb{H}^{0}\left(\Sigma, \mathcal{E} \otimes L_{p}^{k-1}\right) \cong H^{0}\left(L_{\mathcal{E}} \otimes L_{p}^{k-1}\right)$. The first part of the statement follows.

By the above argument we see that $\tilde{E}_{k}^{k}=\left\{\mathcal{E} \in \tilde{E}_{k}: H^{0}\left(\Sigma, L_{\mathcal{E}} \otimes L_{p}^{k-1}\right) \neq\right.$ $0\}$, however $L_{\mathcal{E}}$ is of degree $1-k$, thus $\tilde{E}_{k}^{k}=\left\{\mathcal{E} \in \tilde{E}_{k}: L_{\mathcal{E}}=L_{p}^{1-k}\right\}$, as claimed. This means that for every $\mathcal{E} \in E_{k}$ there is a unique line bundle $L=L_{p}^{1-k} \otimes L_{\mathcal{E}}^{*}$ such that $\mathcal{E} \otimes L \in \tilde{E}_{k}^{k}$. This shows (26).

Remark. By definition $\tilde{\mathcal{N}}_{k}=W_{2,2 k-1}^{0}$ are non-Abelian Brill-Noether loci as defined in [Sun] (cf. [Tei]).

\section{Proof of Theorem 1.1}

In this final section we prove Theorem 1.1.

Proof of Theorem 1.1. The proof proceeds by showing that $\operatorname{ch}_{0}\left(\mathbf{D}_{k}\right)=4 g-4$ then $c_{4 g-3}\left(\mathbf{D}_{k}\right)=0$ and we finish by using Porteous's theorem for $\mathbf{D}_{k}$.

First we make some calculations.

Lemma 8.1 The formal difference of coherent sheaves $\mathbf{D}_{k}$ has rank $4 g-4$, i.e.

$$
\operatorname{ch}_{0}\left(\mathbf{D}_{k}\right)=4 g-4 \text {. }
$$

Moreover

$$
c\left(\mathbf{D}_{k}\right)=\left(1+\frac{\alpha_{\mathcal{M}}}{2}+\frac{\alpha_{\mathcal{M}}^{2}-\beta_{\mathcal{M}}}{4}\right)^{2 g-2}
$$

in the decomposition (5).

Proof. It follows from the long exact sequence (8) that

$$
\mathbf{D}_{k}=-\pi_{\tilde{\mathcal{M}} !}\left(\mathcal{E}_{\tilde{\mathcal{M}}}^{k}\right)=\pi_{\tilde{\mathcal{M}} !}\left(\mathbb{E}_{\mathcal{\mathcal { M }}}^{k} \otimes K_{\Sigma}\right)-\pi_{\tilde{\mathcal{M}} !}\left(\mathbb{E}_{\mathcal{\mathcal { M }}}^{k}\right) .
$$


We can calculate the Chern character of the right hand side by the Grothendieck-Riemann-Roch theorem. This gives

$$
\operatorname{ch}\left(\mathbf{D}_{k}\right)=\pi_{\tilde{\mathcal{M}} *}\left(\operatorname{ch}\left(\mathbb{E}_{\tilde{\mathcal{M}}}^{k}\right)\left(\operatorname{ch}\left(K_{\Sigma}\right)-1\right) \operatorname{td}(\Sigma)\right) .
$$

Now $\operatorname{td}(\Sigma)=1-(g-1) \sigma$ and $\operatorname{ch}\left(K_{\Sigma}\right)=1+(2 g-2) \sigma$. Moreover $\pi_{\tilde{\mathcal{M}} *}$ maps a cohomology class in $a \in H^{*}(\tilde{\mathcal{M}}) \otimes H^{*}(\Sigma)$ of the form

$$
a=a_{0} \otimes 1+\sum_{i=1}^{2 g} a_{1}^{i} \otimes e_{i}+a_{2} \otimes \sigma
$$

to the class $a_{2} \in H^{*}(\tilde{\mathcal{M}})$. The class $a_{2}$ is denoted by $a_{2}=a \backslash \sigma$, while the class $a_{0}$ is denoted by $a_{0}=a \backslash 1$. From this it follows that

$$
\operatorname{ch}\left(\mathbf{D}_{k}\right)=\left(\operatorname{ch}\left(\mathbb{E}_{\tilde{\mathcal{M}}}^{k}\right)((2 g-2) \sigma)(1-(g-1) \sigma)\right) \backslash \sigma=(2 g-2)\left(\operatorname{ch}\left(\mathbb{E}_{\tilde{\mathcal{M}}}^{k}\right) \backslash 1\right) .
$$

Observe that $\operatorname{ch}\left(\mathbb{E}_{\tilde{\mathcal{M}}}^{k}\right) \backslash 1=\operatorname{ch}\left(\left(\mathbb{E}_{\tilde{\mathcal{M}}}^{k}\right)_{p}\right) \in H^{*}(\tilde{\mathcal{M}})$, where $\left(\mathbb{E}_{\tilde{\mathcal{M}}}^{k}\right)_{p}=$ $\left.\mathbb{E}_{\tilde{\mathcal{M}}}^{k}\right|_{\tilde{\mathcal{M}} \times\{p\}}$. It follows from (14) and (15) that $c_{1}\left(\left(\mathbb{E}_{\tilde{\mathcal{M}}}^{k}\right)_{p}\right)=\alpha_{\mathcal{M}}$ and $c_{2}\left(\left(\mathbb{E}_{\tilde{\mathcal{M}}}^{k}\right)_{p}\right)=\left(\alpha_{\mathcal{M}}^{2}-\beta_{\mathcal{M}}\right) / 4$. Hence the formal Chern roots of $\left(\mathbb{E}_{\mathcal{\mathcal { M }}}^{k}\right)_{p}$ are $\left(\alpha_{\mathcal{M}}+\sqrt{\beta_{\mathcal{M}}}\right) / 2$ and $\left(\alpha_{\mathcal{M}}-\sqrt{\beta_{\mathcal{M}}}\right) / 2$. Thus

$$
\begin{aligned}
\operatorname{ch}\left(\left(\mathbb{E}_{\tilde{\mathcal{M}}}^{k}\right)_{p}\right) & =\exp \left(\frac{\alpha_{\mathcal{M}}+\sqrt{\beta_{\mathcal{M}}}}{2}\right)+\exp \left(\frac{\alpha_{\mathcal{M}}-\sqrt{\beta_{\mathcal{M}}}}{2}\right) \\
& =2 e^{\alpha_{\mathcal{M}} / 2} \cosh \left(\sqrt{\beta_{\mathcal{M}}} / 2\right)
\end{aligned}
$$

and hence

$$
\operatorname{ch}\left(\mathbf{D}_{k}\right)=(4 g-4) e^{\alpha_{\mathcal{M}} / 2} \cosh \left(\sqrt{\beta_{\mathcal{M}}} / 2\right) .
$$

This shows that $\operatorname{rank}\left(\mathbf{D}_{k}\right)=\operatorname{ch}_{0}\left(\mathbf{D}_{k}\right)=4 g-4$ and formal calculation gives (27).

(27) has the following immediate corollary:

Corollary $8.2 \quad c_{4 g-3}\left(\mathbf{D}_{k}\right)=0$.

To prove Theorem 1.1 we exhibit $g$ linearly independent elements

$$
r_{0}, r_{1}, . ., r_{g-1} \in H_{c p t}^{6 g-6}(\mathcal{M})
$$

for which $j_{\mathcal{M}}\left(r_{i}\right)=0$. 
To construct $r_{k}$ for $0<k<g$ consider the Zariski open subvarieties

$$
\tilde{\mathcal{M}}_{k}=\tilde{\mathcal{M}} \backslash\left(\tilde{\mathcal{N}} \bigcup_{i=1}^{k-1} \tilde{E}_{i}\right)
$$

and

$$
\mathcal{M}_{k}=\mathcal{M} \backslash\left(\mathcal{N} \bigcup_{i=1}^{k-1} E_{i}\right)
$$

of $\tilde{\mathcal{M}}$ and $\mathcal{M}$ respectively. Restricting the sequence of Theorem 6.2 to $\tilde{\mathcal{M}}_{k}$ yields:

$$
\begin{aligned}
0 & \left.\left.\left.\longrightarrow \mathbb{R}^{0} \pi_{\mathcal{M}_{*}}\left(\mathcal{E}_{\tilde{\mathcal{M}}}^{k}\right)\right|_{\tilde{\mathcal{M}}_{k}} \longrightarrow V\right|_{\tilde{\mathcal{M}}_{k}} \stackrel{\left.f\right|_{\mathcal{\mathcal { M }}_{k}}}{\longrightarrow} W\right|_{\tilde{\mathcal{M}}_{k}} \\
& \left.\longrightarrow \mathbb{R}^{1} \pi_{\mathcal{M}_{*}}\left(\mathcal{E}_{\tilde{\mathcal{M}}}^{k}\right)\right|_{\tilde{\mathcal{M}}_{k}} \longrightarrow 0 .
\end{aligned}
$$

The degeneracy locus of $\left.f\right|_{\tilde{\mathcal{M}}_{k}}$ (where $\left.f\right|_{\tilde{\mathcal{M}}_{k}}$ fails to be an injection) is $D_{k} \cap \tilde{\mathcal{M}}_{k}$ which is $\tilde{E}_{k}^{k}$ from Theorem 7.5. This has codimension $4 g-3$. Furthermore

$$
\begin{aligned}
\operatorname{rank}(W)-\operatorname{rank}(V) & =\operatorname{rank}\left(\mathbb{R}^{1} \pi_{\mathcal{M} *}\left(\mathcal{E}_{\tilde{\mathcal{M}}}^{k}\right)\right)-\operatorname{rank}\left(\mathbb{R}^{0} \pi_{\mathcal{M} *}\left(\mathcal{E}_{\tilde{\mathcal{M}}}^{k}\right)\right) \\
& =\operatorname{rank}\left(\mathbf{D}_{k}\right)=4 g-4
\end{aligned}
$$

by Lemma 8.1. Thus the degeneracy locus has the expected codimension hence we are in the situation of Porteous's theorem (cf. [ACGH]), which gives:

$$
\eta_{\tilde{E}_{k}^{k}}^{\tilde{\mathcal{M}}_{k}}=c_{4 g-3}\left(\left.W\right|_{\tilde{\mathcal{M}}_{k}}-\left.V\right|_{\tilde{\mathcal{M}}_{k}}\right) \in H^{8 g-6}\left(\tilde{\mathcal{M}}_{k}\right) \text {. }
$$

The right hand side equals $c_{4 g-3}\left(\left.\mathbf{D}_{k}\right|_{\tilde{\mathcal{M}}_{k}}\right)$ by (28), which vanishes by Corollary 8.2. Moreover (26) yields

$$
\eta_{\tilde{E}_{k}^{k}}^{\tilde{\mathcal{M}_{k}}} \backslash[\mathcal{J}]=\eta_{E_{k}}^{\mathcal{M}_{k}}
$$

It follows that

$$
\eta_{E_{k}}^{\mathcal{M}_{k}}=0 \in H^{6 g-6}\left(\mathcal{M}_{k}\right)
$$

From now on we work over $\mathcal{M}$. We show by induction on $i$ that there is a formal linear combination

$$
r_{k}^{i}=\sum_{j=k-i}^{k} \lambda_{j} \cdot\left[\eta_{E_{j}}^{\mathcal{M}_{k-i}}\right]
$$


of cohomology classes in $H^{6 g-6}\left(\mathcal{M}_{k-i}\right)$, such that $\lambda_{k}=1$ and the corresponding cohomology class $\sum_{j=k-i}^{k} \lambda_{i} \cdot \eta_{E_{j}}^{\mathcal{M}_{k-i}}$ is 0 in $H^{6 g-6}\left(\mathcal{M}_{k-i}\right)$.

For $i=0$ the statement is just (29). Suppose that there is such formal combination $r_{k}^{i}$. Consider the following bit of the long exact sequence of the pair $\mathcal{M}_{k-i} \subset \mathcal{M}_{k-i-1}$ :

$$
H^{6 g-6}\left(\mathcal{M}_{k-i}, \mathcal{M}_{k-i-1}\right) \longrightarrow H^{6 g-6}\left(\mathcal{M}_{k-i-1}\right) \longrightarrow H^{6 g-6}\left(\mathcal{M}_{k-i}\right) .
$$

Because $\mathcal{M}_{k-i-1} \backslash \mathcal{M}_{k-i}=E_{k-i-1}$ is of real codimension $6 g-6$, the Thom isomorphism transforms this sequence to:

$$
H^{0}\left(E_{k-i-1}\right) \stackrel{\tau}{\longrightarrow} H^{6 g-6}\left(\mathcal{M}_{k-i-1}\right) \stackrel{\rho}{\longrightarrow} H^{6 g-6}\left(\mathcal{M}_{k-i}\right),
$$

where $\tau$ is the Thom map and $\rho$ is restriction. Clearly $\rho\left(\eta_{E_{j}}^{\mathcal{M}_{k-i-1}}\right)=\eta_{E_{j}}^{\mathcal{M}_{k-i}}$. Thus

$$
\rho\left(\sum_{j=k-i}^{k} \lambda_{j} \cdot \eta_{E_{j}}^{\mathcal{M}_{k-i-1}}\right)=\sum_{j=k-i}^{k} \lambda_{j} \cdot \eta_{E_{j}}^{\mathcal{M}_{k-i}}=0 .
$$

The exactness of (30) yields that the cohomology class $\sum_{j=k-i}^{k} \lambda_{j} \cdot \eta_{E_{j}}^{\mathcal{M}_{k-i-1}}$ is in the image of $\tau$. Because $H^{0}\left(E_{k}\right) \cong \mathbb{R}$ there is a real number $-\lambda_{k-i-1} \in \mathbb{R}$ such that

$$
\tau\left(-\lambda_{k-i-1}\right)=\sum_{j=k-i}^{k} \lambda_{j} \cdot \eta_{E_{j}}^{\mathcal{M}_{k-i-1}} \in H^{6 g-6}\left(\mathcal{M}_{k-i-1}\right) .
$$

However a well known property of the Thom map gives $\tau(1)=\eta_{E_{k-i-1}}^{\mathcal{M}_{k-i-1}}$, thus from (31) the formal linear combination

$$
r_{k}^{i+1}=\sum_{j=k-i-1}^{k} \lambda_{j} \cdot\left[\eta_{E_{j}}^{\mathcal{M}_{k-i-1}}\right]
$$

is 0 , when considered as a class in $H^{6 g-6}\left(\mathcal{M}_{k-i-1}\right)$. This proves the existence of formal linear combinations $r_{k}^{i}$ for all $0 \leq i \leq k-1$.

Using $r_{k}^{k-1}$ an identical argument gives the formal linear combination

$$
r_{k}^{\prime}=\lambda \cdot\left[\eta_{\mathcal{N}}^{\mathcal{M}}\right]+\sum_{j=1}^{k} \lambda_{j} \cdot\left[\eta_{E_{j}}^{\mathcal{M}}\right]
$$

with the property that $\lambda_{k}=1$ and $r_{k}^{\prime}$ when considered as an element of $H^{6 g-6}(\mathcal{M})$ is 0 . Now the compactly supported cohomology class

$$
r_{k}=\lambda \cdot \bar{\eta}_{\mathcal{N}}^{\mathcal{M}}+\sum_{j=1}^{k} \lambda_{j} \cdot \bar{\eta}_{E_{j}}^{\mathcal{M}} \in H_{c p t}^{6 g-6}(\mathcal{M})
$$


has the property that $j_{\mathcal{M}}\left(r_{k}\right)=r_{k}^{\prime}=0$, where by abuse of notation $r_{k}^{\prime}$ denotes the cohomology class in $H^{6 g-6}(\mathcal{M})$ corresponding to the formal linear combination $r_{k}^{\prime}$.

We have found $g-1$ linearly independent compactly supported cohomology classes $r_{1}, . ., r_{g-1} \in H_{c p t}^{6 g-6}(\mathcal{M})$. Clearly $\bar{\eta}_{\mathcal{N}}^{\mathcal{M}}$ is not in the span of $r_{1}, . ., r_{g-1}$. Moreover for each $0<i<g$ we have

$$
\int_{\mathcal{M}} \bar{\eta}_{\mathcal{N}}^{\mathcal{M}} \wedge r_{i}=0
$$

since $j_{\mathcal{M}}\left(r_{i}\right)=0$. Furthermore

$$
\int_{\mathcal{M}} \bar{\eta}_{\mathcal{N}}^{\mathcal{M}} \wedge \bar{\eta}_{\mathcal{N}}^{\mathcal{M}}=\int_{\mathcal{N}} c_{3 g-3}\left(T_{\mathcal{N}}^{*}\right)=0 .
$$

Thus $\bar{\eta}_{\mathcal{N}}^{\mathcal{M}}$ is perpendicular to $r_{1}, . ., r_{g-1}$ and $\bar{\eta}_{\mathcal{N}}^{\mathcal{M}}$, which constitutes a basis for $H_{c p t}^{6 g-6}(\mathcal{M})$, and so $j_{\mathcal{M}}\left(\bar{\eta}_{\mathcal{N}}^{\mathcal{M}}\right)=0$.

Putting our findings together: we have $g$ linearly independent middle dimensional compactly supported classes $r_{0}=\bar{\eta}_{\mathcal{N}}^{\mathcal{M}}$ and $r_{1}, . ., r_{g-1}$ in the kernel of the forgetful map $j_{\mathcal{M}}: H_{c p t}^{6 g-6}(\mathcal{M}) \rightarrow H^{6 g-6}(\mathcal{M})$.

Theorem 1.1 is finally proved.

\section{References}

[ACGH] E. Arbarello, M. Cornalba, P.A. Griffiths, J. Harris, Geometry of algebraic curves $I$ Springer, Berlin, 1985

[At,Bo] M. Atiyah, R. Bott. Yang-Mills equations over Riemann surfaces, Philos. Trans. Roy. Soc. London Series A, 308 (1982) 523-615

[At,Hi] M. Atiyah, N. Hitchin. The geometry and dynamics of magnetic monopoles, Princeton University Press, 1987

[Bar] V. Baranovsky, Cohomology ring of the moduli space of stable vector bundles with odd determinant, Izv. Russ. Acad. Nauk 58/4 (1994) 204-210

[BJSV] M. Bershadsky, A. Johansen, V. Shadov, C. Vafa. Topological reduction of 4D SYM to 2D $\sigma$-models. Nuclear Phys. B 448 (1995), no. $1-2,166-186$.

[Bi,Ra] I. Biswas, S. Ramanan. An infinitesimal study of the moduli space of Hitchin pairs, J. London Math. Soc. 49 (1994), 219-231 
[DeRh] De Rham. Differential manifolds. English edition, Berlin, Heidelberg, New York: Springer, 1984

[Dod] J. Dodziuk. Vanishing theorems for square integrable harmonic forms, Proc. Indian. Acad. Sci. Math. Sci. 90 (1981), 21-27.

[Dr,Na] J.-M. Drezet, M.S. Narasimhan, Groupe Picard des variétés de modules de fibrés semistables sur les courbes algébraiques, Invent. Math. 97 53-94 (1989)

[Gr,Ha] P. Griffiths, J. Harris. Principles of algebraic geometry. New York, Wiley 1978

[Gun] R.C. Gunning, Lectures on vector bundles over Riemann surfaces Princeton University Press, 1967.

[Ha,Na] G. Harder, M.S. Narasimhan. On the cohomology groups of moduli spaces of vector bundles over curves. Math. Ann. 212, 215-248

[Har] R. Hartshorne, Algebraic geometry Springer-Verlag, New York, 1977

[Hau1] T. Hausel. Compactification of moduli of Higgs bundles, J. Reine Angew. Math.503 (1998),169-182.

[Hau2] T. Hausel. Geometry of the moduli space of Higgs bundles, Ph.D. thesis, 1998, Cambridge

[Ha,Th] T. Hausel, M. Thaddeus, Cohomology ring of the moduli space of Higgs bundles, under preparation

[Hit1] N. Hitchin. The self-duality equations on a Riemann surface, Proc. London Math. Soc. (3) 55 (1987) 59-126.

[Hit2] N. Hitchin. private communication

[HKLR] N. Hitchin, A. Karlhede, U. Lindstrom, M. Roček, Hyper-Kähler metrics and supersymmetry, Comm. Math. Phys. 108 (1987), no.4, $535-589$

[Hu,Le] D. Huybrechts, M. Lehn. The geometry of moduli spaces of sheaves, Friedr. Vieweg und Sohn, Braunschweig, 1997

[Ki,Ne] A.D. King, P.E. Newstead, On the cohomology ring of the moduli space of rank 2 vector bundles on a curve, Topology 37 No.2 (1998) $407-418$ 
[Kir] F. Kirwan. The cohomology ring of moduli space of bundles over Riemann surfaces. J. Amer. Math. Soc. 5 (1992), no.4, 853-906

[Na,Se] M.S. Narasimhan, C.S. Seshadri. Stable and unitary vector bundles on a compact Riemann surface, Ann. Math. (1965) 540-567

[New] P.E. Newstead. Introduction to moduli problems and orbit spaces, Tata Inst. Bombay, 1978

[Nit] N. Nitsure. Moduli space of semistable pairs on a curve. Proc. London Math. Soc. (3) 62 (1991), 275-300

[Se,Se] G. Segal, A. Selby. Cohomology of the space of magnetic mono -poles, 775-787, Comm. Math. Phys. 177, 1996

[Sen] A. Sen. Dyon-monopole bound states, self-dual harmonic forms on the multi-monopole moduli space, and $S L(2, \mathbb{Z})$ invariance in string theory. Phys.-Lett.-B 329 (1994), no.2-3,217-221.

[Si,Ti] B.Siebert, G.Tian: Recursive relations for the cohomology ring of moduli space of stable bundles, Turkish J. Math. 19 (1996) 131-144

[Sim] C. Simpson. Higgs bundles and local systems. Publ. Math. I.H.E.S. 75 (1992), 5-95

[Sun] N. Sundaram. Special divisors and vector bundles. Tohoku Math. $J$. (2)39 (1987), no.2, 175-213

[Tei] M. Teixidor i Bigas, Brill-Noether theory for stable vector bundles, Duke Math. J. 62 (1991), no.2,385-400

[Tha1] M. Thaddeus. Topology of the moduli space of stable bundles over a compact Riemann surface, 1990, unpublished

[Tha2] M. Thaddeus. An introduction to the topology of the moduli space of stable bundles on a Riemann surface, Collection: Geometry and Physics (Aarhus, 1995), 71-99

[Tha3] M. Thaddeus. Stable pairs, linear systems and the Verlinde formula, Invent. Math. 117 (1994) 317-353

[Zag] D. Zagier. On the cohomology of moduli spaces of rank 2 vector bundles over curves, The moduli space of curves, ed. R. Dijkgraaf, C. Faber and G. van der Geer, Progress in Mathematics 129, Birkhäuser, 1995 